\title{
pEPito-driven PEDF expression ameliorates Diabetic Retinopathy hallmarks
}

\author{
Sofia M. Calado ${ }^{1,2}$, Francisco Diaz-Corrales ${ }^{3}$, Gabriela A. Silva $^{2 \#}$ \\ ${ }^{1}$ Doctoral Program in Biomedical Sciences, Department of Biomedical Sciences and \\ Medicine, University of Algarve, 8005-139 Faro, Portugal \\ ${ }^{2}$ CEDOC, NOVA Medical School | Faculdade de Ciências Médicas, Universidade Nova de \\ Lisboa, Campo Mártires da Pátria 130, 1169-056 Lisboa, Portugal \\ ${ }^{3}$ Department of Cell Therapy and Regenerative Medicine, Andalusian Molecular Biology and \\ Regenerative Medicine Centre (CABIMER), Avda. Americo Vespucio, Seville 41092, Spain
}

\section{\# Corresponding author:}

Gabriela A. Silva

Email: gabriela.silva@nms.unl.pt

Phone: +35121880 3109

Fax: +351218803010 


\section{Abstract}

Diabetic retinopathy (DR) is one of the major complications of Diabetes mellitus. It is characterized by retinal microvascular changes caused by chronic exposure to hyperglycemia, leading to low tissue oxygenation and ultimately to neovascularization. Laser photocoagulation and vitrectomy are the most efficient treatments for DR, but display severe side effects such as the destruction of the healthy retina. Another clinical approach uses anti-angiogenic agents to prevent and delay progression of neovascularization, but these require recurrent local administrations which increase the possibility of retinal detachment, vitreous hemorrhage, and cataract formation.

Studies in human diabetic retinas have revealed an imbalance between pro-angiogenic factors such as the vascular endothelial growth factor (VEGF) and anti-angiogenic factors, like pigment epithelial-derived factor (PEDF). This imbalance favors pathological angiogenesis contributing to DR, and can constitute a therapeutic target. Gene therapy was recently shown to be an adequate intervention for long-term treatment of several retinal pathologies. We have previously shown the newly engineered episomal vector pEPito to be able of sustained gene expression in the mouse retina. We here show that pEPito was able to overexpress PEDF for up to three months, both in in vitro cultures of human RPE cells, as well as in the retina of diabetic mice after a single subretinal injection. In vivo, in parallel with the increase in PEDF we observed a decrease in VEGF levels in injected compared with non-injected eyes and a significant effect on two hallmarks of DR: reduction of glucose transport (by glucose transporter GLUT1), and reduction of inflammation by decreased reactivity of microglia. Jointly, these results point to a significant therapeutic potential of gene therapy with $p E P i t o-P E D F$ for the treatment of DR.

Key words: Gene therapy; Diabetic Retinopathy; pEPito; PEDF; microglia; 


\section{Introduction}

Diabetes mellitus (DM) is a group of metabolic diseases characterized by high blood glucose that leads to several complications, including diabetic retinopathy (DR). DR is a progressive disease that features microvascular dysfunction due to chronic exposure to hyperglycemia. ${ }^{1}$ Hyperglycemia is associated with a wide-range of biochemical events that contribute for the progression of the disease, such as accumulation of sorbitol, advanced glycation endproducts (AGE), oxidative stress, protein kinase $C$ activation, inflammation, up-regulation of the renin-angiotensin system and vascular endothelial growth factor (VEGF). ${ }^{1}$ Altogether, these mechanisms contribute to physiological changes in the retina, including capillary basement membrane thickening that induces pericyte and endothelial cell death, causing inner blood retinal barrier (BRB) breakdown. This loss of retinal capillary function leads to vascular wall leakage, inflammation and ischemia, contributing to retinal neovascularization, formation of microaneurysms, edema, and hemorrhages, which lead to irreversible blindness. $^{1-3}$

Attempts to revert pathological neovascularization have focused on the inhibition of VEGF, since the increase in its levels causes changes in the BRB, resulting in retinal macular edema and neovascularization. ${ }^{3}$ However, altered VEGF levels are not the single event associated with neovascularization: during DR, the balance between pro- and antiangiogenic factors that inhibits pathological angiogenesis.is disrupted. This is caused by an up-regulation of angiogenic VEGF and down-regulation of the anti-angiogenic and neurotrophic factor pigment epithelial-derived factor (PEDF), secreted by retinal pigment epithelial (RPE) cells. ${ }^{4}, 5$ PEDF has been shown to reduce neovascularization when administered as a protein to a mouse model of neovascularization. ${ }^{6,7}$

Common treatments for DR are laser photocoagulation and vitrectomy; they can however cause retinal damage and permanent impaired vision, with the success of the treatment highly influenced by the disease progression stage. ${ }^{8}$ Recently, anti-VEGF agents, such as pegaptanib, ranibizumab and bevacizumab, have been shown to be more effective inhibiting 
NV than surgical approaches. The major disadvantage of these agents is their short half-life, which requires repeated intravitreal injections in order to obtain a sustained therapeutic effect. $^{5-11}$ To achieve a sustained therapeutic effect, one can turn to gene therapy, which has already been tested as a treatment for several retinal pathologies, ${ }^{12-14}$ including ocular neovascularization. ${ }^{15}$ Viral vectors, with overall limitations such limited size of the inserted gene, immune response, toxicity, and possible oncogenicity, are widely used for gene therapy. Alternative approaches encompass both non-viral vehicles and expression systems that can efficiently deliver and provide sustained gene expression. We have previously described the effectiveness of the self-replicating episomal vector, pEPito, in long-term gene expression in the mouse retina. ${ }^{16}$ Based on this study, we have used pEPito as an expression vector for PEDF to assess its potential for treatment of diabetic retinopathy. Using a two-fold approach - in vitro using human RPE cells and in vivo, using the Ins2 Akita mouse model of DR - we aimed to determine if PEDF can constitute a therapeutic alternative for diabetic retinopathy. 


\section{Materials and Methods}

\section{Vector construction}

pEPito-hCMV-eGFP-IRES-IB containing the human CMV enhancer/human elongation factor 1 alpha promoter was used as backbone, as described before. ${ }^{16,17}$ Human PEDF was amplified with specific primers containing Nhe I (5') and Bgl II (3') restriction sites. The amplified fragment was digested with Nhe I and $\mathrm{Bgl}$ II and cloned in the pEPito-hCMV, digested with the same enzymes. The resulting construct was confirmed by restriction enzyme digestion and sequencing. The new plasmid pEPito-hCMV-PEDF with a $6.2 \mathrm{~kb}$ size was propagated in E. coli GT115.

\section{Evaluation of PEDF expression driven by the $p E P$ ito vector in vitro} Human retinal pigment epithelial (RPE) cell line D407, used in the in vitro experiments, was kindly provided by Dr. Jean Bennett from the University of Pennsylvania (USA). Cells were kept in culture at $37^{\circ} \mathrm{C}$ in a humid chamber with $5 \% \mathrm{CO}_{2}$ and were grown in Dulbecco's Modified Eagle's Medium (DMEM) (Sigma-Aldrich, USA) supplemented with 1\% Penicillin/Streptomycin (Sigma-Aldrich), 1\% glutamine (Sigma-Aldrich) and 5\% foetal bovine serum (Sigma-Aldrich). Culture medium was changed every 2 days.

Transfection was performed using FuGENE ${ }^{\circledR}$ HD (Promega) with a $3: 1$ ( $\mu \mathrm{L}$ of FuGENE ${ }^{\circledR}$ $\mathrm{HD}: \mu \mathrm{g}$ of DNA) ratio, according to the manufacturer's instructions. Briefly, $2 \times 10^{5}$ cells were seeded in a 6-well tissue culture plate (Orange Scientific) and $24 \mathrm{~h}$ after seeding, cells were transfected with $1 \mu \mathrm{g}$ of DNA. $24 \mathrm{~h}$ post transfection cells were transferred from the 6 -well tissue culture plate to $6 \mathrm{~cm}$ Petri dishes. Once a week, for up to three months, the cells were subcultured and the samples of cells and culture medium collected for Western blot analysis. 
Four-month old Ins2 ${ }^{\text {Akita }}$ (diabetic) mice (The Jackson Laboratory) housed under controlled temperature and a $12 \mathrm{~h} \mathrm{light/dark} \mathrm{cycle} \mathrm{with} \mathrm{food} \mathrm{and} \mathrm{water} \mathrm{ad} \mathrm{libitum} \mathrm{were} \mathrm{used} \mathrm{for} \mathrm{the} \mathrm{in}$ vivo experiments. Diabetic phenotype was confirmed 2-months after birth by measuring blood glucose levels from a tail puncture (Freestyle Precision, Abbot), with animals used in this study exhibiting blood glucose $\geq 500 \mathrm{mg} / \mathrm{dl}$. All experimental procedures were carried out according to the Portuguese and European Laboratory Animal Science Association (FELASA) Guide for the Care and Use of Laboratory Animals, the European Union Council Directive 2010/63/EU for the use of animals in research and the Association for Research in Vision and Ophthalmology (ARVO) for the use of animals in ophthalmic and vision research. Mice were profoundly anesthetized by intraperitoneal injection of Avertin ${ }^{\oplus}$. 1uL of DNA (1 $\mathrm{ug} / \mathrm{uL}$ ) was injected in the subretinal space of the left eye by using an automatic pump injector (WPI) and electroporated using $7 \mathrm{~mm}$ tweezer electrodes (tweezertodes, Harvard Apparatus) connected to a BTX ECM 830 (Harvard Apparatus), as previously described. ${ }^{18}$ At 3-months post injection the animals were humanely sacrificed by cervical dislocation, the eyes enucleated, and processed depending on the experiment.

\section{Immunofluorescence for PEDF expression in vivo}

For immunohistochemistry, eyes were fixed with ice-cold $4 \%$ paraformaldehyde (4\% PFA) in PBS overnight at $4{ }^{\circ} \mathrm{C}$, cryoprotected in $30 \%$ sucrose in PBS and embedded in OCT mounting medium (Tissue-Tek). Immunohistochemistry was performed either in $10 \mu \mathrm{m}$ thick serial sections or whole retinas. Briefly, the samples were washed in $0.1 \%$ Triton X100/PBS and blocked in $1 \%$ goat serum/PBS at room temperature for $1 \mathrm{~h}$. Incubation with the primary antibodies - rabbit polyclonal anti-PEDF 1:100; (Merck Millipore) and rabbit polyclonal antiIba1 (1:500; Wako) - was performed overnight at $4^{\circ} \mathrm{C}$.

After incubation samples were washed 3 times in $0.1 \%$ Triton X100/PBS, and incubated with the secondary antibody (Alexa Fluor ${ }^{\circledR}$ 594; 1:500; Life Technologies, USA) at room temperature for $1 \mathrm{~h}$. After 3 washes, slides were mounted with Fluoromount G (SouthernBiotech, USA) containing 4',6'-diamidino-2-phenylindole (DAPI). Images were 
obtained using an AxioVision microscope, using appropriate filter sets (Axio Observer Z2, Zeiss).

\section{PEDF expression by Western blot analysis}

Since PEDF is a secreted protein and PEDF-pEPito administration was performed subretinally, we evaluated its expression by Western blot in whole retinas and RPE cells. The latter were isolated by dissection of the eyeball and homogenized in ice-cold RIPA buffer (50 mM Tris-HCl pH 7.4, 1\% NP-40, 0.25\% Na-deoxycholate, $150 \mathrm{mM} \mathrm{NaCl}$, and 1 mM EDTA) containing a protease inhibitor cocktail (Roche, Germany).

Whole protein extract of D407 cells was also prepared using cold RIPA buffer supplemented with protease inhibitor cocktail. Protein content was measured by the Bradford assay and samples stored at $-80^{\circ} \mathrm{C}$. Thirty micrograms of protein extract were separated in a denaturing $12 \%$ SDS-PAGE gel, the proteins transferred to a PVDF membrane (Amersham, UK), and blocked using Superblock Blocking buffer (Thermo Scientific) containing $0.1 \%$ of Tween-20 (Sigma-Aldrich, USA) for $1 \mathrm{~h}$ at room temperature. The primary antibodies used were rabbit polyclonal anti-PEDF (1:1000; Merck Millipore), rabbit polyclonal anti-VEGF (1:1000; Abcam), rabbit polyclonal anti-GLUT1 (1:3000; Abcam), goat polyclonal anti-lba1 (1:1000; Sigma-Aldrich), and rabbit polyclonal anti-GFAP (1:1000; Abcam), and were incubated overnight at $4{ }^{\circ} \mathrm{C}$. $\beta$-Actin was incubated for $1 \mathrm{~h}$ at room temperature $(1: 10000$; Sigma-Aldrich). The membrane was probed with an HRP-conjugated secondary antibody for $1 \mathrm{~h}$ at room temperature and the immunoreactive bands were detected by chemiluminescence, using an ECL Plus kit (Amersham).

Secreted PEDF was isolated from the culture medium by protein precipitation using 4 volumes of ice-cold acetone for $1 \mathrm{~h}$, at $-20^{\circ} \mathrm{C}$. After precipitation samples were centrifuged for 10 minutes at $13000 \mathrm{~g}$, the supernatant decanted, and the pellet air-dried for 30 minutes at room temperature. The pellet containing precipitated proteins was then re-suspended in $1 \mathrm{x}$ sample buffer and analyzed by Western blot. 


\section{Statistical analysis}

All experiments were performed in triplicate and the results expressed as mean \pm standard error of the mean (SEM). Statistical analysis was performed with GraphPad Prism software. $A$ value of $P<0.05$ was considered to be statistically significant. 


\section{Results}

\section{Characterization of PEDF in the retina of Ins2 ${ }^{\text {Akita }}$ diabetic mice}

Clinical studies have demonstrated a negative correlation between PEDF levels and the development of proliferative diabetic retinopathy. ${ }^{19}$ To establish if Ins2 $2^{\text {Akita }}$ diabetic mice mimic what was observed in humans and are a valid animal model to evaluate PEDF effects, PEDF expression was evaluated in the retina of 4-month old Ins2 $2^{\text {Akita }}$ diabetic and compared to age-matched wild-type mice. As can be observed in figures $1 A$ and $B$, there is a significantly decrease in PEDF expression in the retina of diabetic animals compared with wild-type ones, similar to what is observed in humans. This decrease is much more evident in the retinal pigment epithelium (RPE) of diabetic mice (1C and D), compared with expression in the neural retina.

\section{pEPito enables long-term expression of PEDF in mitotic RPE cells}

For an efficient gene therapy strategy, the expression vector should be able of long-term and sustained levels of transgene expression. In our previous study we have described the effectiveness of the pEPito episomal vectors in sustained gene expression in retinal cells. ${ }^{16}$ We have also shown that plasmids containing the humanized CMV promoter (hCMV), known to be less affected by epigenetic silencing effects, were more efficient in promoting gene expression in retinal cells both in vitro and in vivo. ${ }^{16}$ Based on these results, we have chosen the pEPito-hCMV backbone to clone PEDF. We have found that our new construct was able to overexpress PEDF for almost three months in actively dividing human RPE cells (figure 2).

\section{Restoration of PEDF protein levels in the retina of Ins2 ${ }^{\text {Akita }}$ mice}

Subretinal injection of a $1 \mu \mathrm{L}$ solution of pEPito-hCMV-PEDF was performed into one eye of 4-month old Ins2 $2^{\text {Akita }}$ mice, with the contralateral eye remaining non-injected, as a control. 
Immediately after injection the eyes were electroporated to facilitate entry of the expression vector into the cell. ${ }^{18}$ After the procedure and until sacrifice of the animal, the injected eyes had a normal appearance and absence of inflammation, similar to the non-injected, contralateral eye.

The effect of PEDF overexpression was evaluated in the retina of the Ins2 ${ }^{\text {Akita }}$ diabetic mouse, a model of DR. Three months post injection, PEDF expression was analyzed in 10 micron eye sections, and immunohistochemistry shows an increase in PEDF detected in the injected eye, when compared with the contralateral non-injected one. Moreover, the intensity of PEDF expression was comparable to one observed in the WT mice (figure 3).

These results were confirmed by Western blot, with an increase in PEDF expression in injected compared with non-injected eyes. Moreover, the overexpression of PEDF in the retina of Ins $2^{\text {Akita }}$ diabetic mice was also associated with a decrease in other hallmarks of diabetic retinopathy, such as GFAP, VEGF and glucose transporter 1 (GLUT1) in the retina. The last two were also reduced in the RPE of the injected animals, as observed in figure 4. One of the features observed in the Ins $2^{\text {Akita }}$ diabetic mouse is the activation of microglia evidenced by retracted and swollen projections, ${ }^{20}$ which correlates with inflammation in the retina. To analyze for microglia activation as a marker of inflammation, we have performed immunohistochemistry for Iba1 (ionized calcium-binding adaptor molecule, figure 5), specific for microglia and macrophages. ${ }^{20}$ When compared with injected retinas (panel B), the microglia of non-injected retinas (panel A) has more retracted and swollen projections, a feature of reactive morphology of microglia. Iba1 expression is decreased in the injected eyes (panel D) when compared with non-injected (panel C), indicating that overexpression of PEDF has anti-inflammatory properties. 


\section{Discussion}

Diabetic Retinopathy is a severely handicapping disease that affects $90 \%$ of type 1 DM patients and $60 \%$ of type $2 \mathrm{DM}$ patients, ${ }^{21}$ and the leading cause of blindness in developed countries. Neovascularization has been associated with an imbalance between VEGF and PEDF. ${ }^{5}$ Targeting this imbalance can constitute an innovative therapeutic approach, but to be an effective therapeutic strategy this balance should be restored in the long-term, and gene therapy can achieve long-term expression. Our group has recently shown the effectiveness of episomal pEPito expression systems in long-term transgene expression in the mouse retina. ${ }^{16}$ Based on these previous results in this study we have cloned the PEDF gene into the pEPito-hCMV backbone and tested this vector both in vitro in human RPE cells, and in vivo in the Ins2 $2^{\text {Akita }}$ mouse. The Ins $2^{\text {Akita }}$ mouse is a spontaneous non-obese model of type 1 diabetes used to study $\mathrm{DR}^{22-24}$ In these animals retinal complications including vascular leakage, loss of pericytes, inflammation, thickening of the inner retinal layers, ${ }^{22}$ and increase of angiogenic markers are visible approximately 12 weeks after the onset of hyperglycemia. ${ }^{24}$

To test the therapeutic potential of our approach, it was necessary to assess if the PEDF expression profile in diabetic Ins2Akita mice reproduces what is observed in humans. Our results show a significant decrease in PEDF expression levels in the inner retina of the Ins2Akita mice compared with age-matched WT controls (figure 1A-B). This decrease was even more pronounced in the RPE of the diabetic mice (Figure 1C-D) showing that the secretion of PEDF by the retinal pigment epithelium (RPE) is affected in the early stages of the disease. This correlates well to what was described for humans. ${ }^{25,26}$

Our in vitro results show that pEPito was able to overexpress PEDF for up to three months in mitotically active RPE cells, proving the mitotic stability of this episomal vector and its longterm expression. In the Ins $2^{\text {Akita }}$ diabetic mouse our expression system was able to express PEDF for up to three months. As expected, PEDF overexpression was detected in the RPE layer of the retina of injected eyes, where the plasmid was delivered, and in the inner retina (figure 3), since PEDF is a secreted protein. Since the eye is an immune-privileged organ, 
possessing two distinct blood-retinal barriers that limit the passage of substances between the eye and systemic circulation, the leakage of PEDF is not a concern.

It was previously described that angiogenic VEGF is overexpressed in the retina of 6-month old Ins2 ${ }^{\text {Akita }}$ mice. ${ }^{24}$ Moreover, it was also described that intravitreal injection of PEDF was responsible for down-regulation of VEGF and inhibition of VEGF-VEGFR2 binding in the retina of the oxygen-induced retinopathy mouse model. ${ }^{26}$ Similarly, our results show that overexpression of PEDF in the retina of Ins2 ${ }^{\text {Akita }}$ mice markedly decreases the expression of VEGF (figure 4), compared with non-injected eyes, where VEGF levels are higher.

Recent studies suggest that changes in the microglia, such as increased reactivity, are the early feature of $\mathrm{DR} .{ }^{25} \operatorname{In}$ Ins2 $2^{\text {Akita }}$ diabetic mice, as early as 8 weeks after the onset of hyperglycemia $^{22}$ there is an increase in the reactivity of retinal microglia, a feature of the inflammatory status of the retina. ${ }^{25}$ After injection of the pEPito-hCMV-PEDF we have found that overexpression of PEDF strongly reduces microglia activity, visualized by the nonreactive morphology of microglia. In contrast, in the non-injected eye the reactive morphology remained unchanged (figure 5). These results are in accordance with a previous study in which topical administration of PEDF peptide (PEDF78-121) in Ins2 ${ }^{\text {Akita }}$ mice prevented microglia activation. ${ }^{7}$ We have also found a decrease in GFAP expression in the injected eye, compared with the non-injected one (figure 4). GFAP expression by astrocytes, Müller and glial cells increases after retinal injury such as ischemia, glaucoma, ${ }^{27}$ and diabetes. ${ }^{28}$ Our results show that treatment with PEDF decreases GFAP expression, which can be associated with a reversal in the retinal injury.

Overall, this study demonstrates the beneficial effect of long-term expression of PEDF in the retina of diabetic mice, through the anti-inflammatory and anti-angiogenic potential of PEDF in preventing retinal complications of diabetes. 


\section{Acknowledgements}

We would like to acknowledge Dr. Rudolf Haase for kindly providing the pEPito backbone for our experiments.

This work was supported by the Portuguese Foundation for Science and Technology (FCT) with individual grants to S.M. Calado (SFRH/BD/76873/2011), funding to G.A. Silva (EXPL-BIM-MEC-1433-2013) and Pest-OE/EQB/LA0023/2013. G.A. Silva was also funded by PIRG05-GA-2009-249314-EyeSee.

iNOVA4Health - UID/Multi/04462/2013, a program financially supported by Fundação para a Ciência e Tecnologia / Ministério da Educação e Ciência, through national funds and co-funded by FEDER under the PT2020 Partnership Agreement is acknowledged

\section{Author Discloser Statement}

No competing financial interests exist. 


\section{References}

1. Cheung, N., Mitchell, P., and Wong, T.Y., Diabetic retinopathy. Lancet, 2010. 376(9735):

p. 124-36.

2. Frank, R., Diabetic retinopathy. Progress in Retinal and Eye Research, 1995. 14(2): p. 31.

3. Chiu, C.J. and Taylor, A., Dietary hyperglycemia, glycemic index and metabolic retinal diseases. Prog Retin Eye Res, 2011. 30(1): p. 18-53.

4. Broadhead, M.L., Becerra, S.P., Choong, P.F., et al., The applied biochemistry of PEDF and implications for tissue homeostasis. Growth Factors, 2010. 28(4): p. 280-5.

5. Farjo, K.M. and Ma, J.X., The potential of nanomedicine therapies to treat neovascular disease in the retina. J Angiogenes Res, 2010. 2: p. 21.

6. Yoshida, Y., Yamagishi, S., Matsui, T., et al., Protective role of pigment epithelium-derived factor (PEDF) in early phase of experimental diabetic retinopathy. Diabetes Metab Res Rev, 2009. 25(7): p. 678-86.

7. Liu, Y., Leo, L.F., McGregor, C., et al., Pigment epithelium-derived factor (PEDF) peptide eye drops reduce inflammation, cell death and vascular leakage in diabetic retinopathy in Ins2(Akita) mice. Mol Med, 2012. 18: p. 1387-401.

8. Fong, D.S., Aiello, L.P., Ferris, F.L., 3rd, et al., Diabetic retinopathy. Diabetes Care, 2004. 27(10): p. 2540-53.

9. Bakri, S.J., Snyder, M.R., Reid, J.M., et al., Pharmacokinetics of intravitreal ranibizumab (Lucentis). Ophthalmology, 2007. 114(12): p. 2179-82.

10. Praidou, A., Androudi, S., Brazitikos, P., et al., Angiogenic growth factors and their inhibitors in diabetic retinopathy. Curr Diabetes Rev, 2010. 6(5): p. 304-12.

11. Vinores, S.A., Pegaptanib in the treatment of wet, age-related macular degeneration. Int J Nanomedicine, 2006. 1(3): p. 263-8.

12. Maguire, A.M., High, K.A., Auricchio, A., et al., Age-dependent effects of RPE65 gene therapy for Leber's congenital amaurosis: a phase 1 dose-escalation trial. Lancet, 2009. 374(9701): p. 1597-605. 
13. MacLaren, R.E., Groppe, M., Barnard, A.R., et al., Retinal gene therapy in patients with choroideremia: initial findings from a phase 1/2 clinical trial. Lancet, 2014. 383(9923): p. 1129-37.

14. Bainbridge, J.W., Smith, A.J., Barker, S.S., et al., Effect of gene therapy on visual function in Leber's congenital amaurosis. N Engl J Med, 2008. 358(21): p. 2231-9.

15. Igarashi, T., Miyake, K., Kato, K., et al., Lentivirus-mediated expression of angiostatin efficiently inhibits neovascularization in a murine proliferative retinopathy model. Gene Ther, 2003. 10(3): p. 219-26.

16. Calado, S.M., Oliveira, A.V., Machado, S., et al., Sustained gene expression in the retina by improved episomal vectors. Tissue Eng Part A, 2014. 20(19-20): p. 2692-8.

17. Haase, R., Argyros, O., Wong, S.P., et al., pEPito: a significantly improved non-viral episomal expression vector for mammalian cells. BMC Biotechnol, 2010. 10: p. 20.

18. Matsuda, T. and Cepko, C.L., Electroporation and RNA interference in the rodent retina in vivo and in vitro. Proc Natl Acad Sci U S A, 2004. 101(1): p. 16-22.

19. Zhu, X.F. and Zou, H.D., PEDF in diabetic retinopathy: a protective effect of oxidative stress. J Biomed Biotechnol, 2012. 2012: p. 580687.

20. Zhu, D., Wu, J., Spee, C., et al., BMP4 mediates oxidative stress-induced retinal pigment epithelial cell senescence and is overexpressed in age-related macular degeneration. J Biol Chem, 2009. 284(14): p. 9529-39.

21. Garg, S. and Davis, R.M., Diabetic Retinopathy Screening Update. Clinical Diabetes, 2009. 27(4): p. 140-145.

22. Barber, A.J., Antonetti, D.A., Kern, T.S., et al., The Ins2Akita mouse as a model of early retinal complications in diabetes. Invest Ophthalmol Vis Sci, 2005. 46(6): p. 2210-8.

23. Gastinger, M.J., Singh, R.S., and Barber, A.J., Loss of cholinergic and dopaminergic amacrine cells in streptozotocin-diabetic rat and Ins2Akita-diabetic mouse retinas. Invest Ophthalmol Vis Sci, 2006. 47(7): p. 3143-50.

24. Han, Z., Guo, J., Conley, S.M., et al., Retinal angiogenesis in the Ins2(Akita) mouse model of diabetic retinopathy. Invest Ophthalmol Vis Sci, 2013. 54(1): p. 574-84. 
25. Grigsby, J.G., Cardona, S.M., Pouw, C.E., et al., The role of microglia in diabetic retinopathy. J Ophthalmol, 2014. 2014: p. 705783.

26. Zhang, S.X., Wang, J.J., Gao, G., et al., Pigment epithelium-derived factor downregulates vascular endothelial growth factor (VEGF) expression and inhibits VEGFVEGF receptor 2 binding in diabetic retinopathy. J Mol Endocrinol, 2006. 37(1): p. 1-12.

27. Chang, M.L., Wu, C.H., Jiang-Shieh, Y.F., et al., Reactive changes of retinal astrocytes and Muller glial cells in kainate-induced neuroexcitotoxicity. J Anat, 2007. 210(1): p. 54-65.

28. Barber, A.J., Antonetti, D.A., and Gardner, T.W., Altered expression of retinal occludin and glial fibrillary acidic protein in experimental diabetes. The Penn State Retina Research Group. Invest Ophthalmol Vis Sci, 2000. 41(11): p. 3561-8.

\section{Legends}


Figure 1

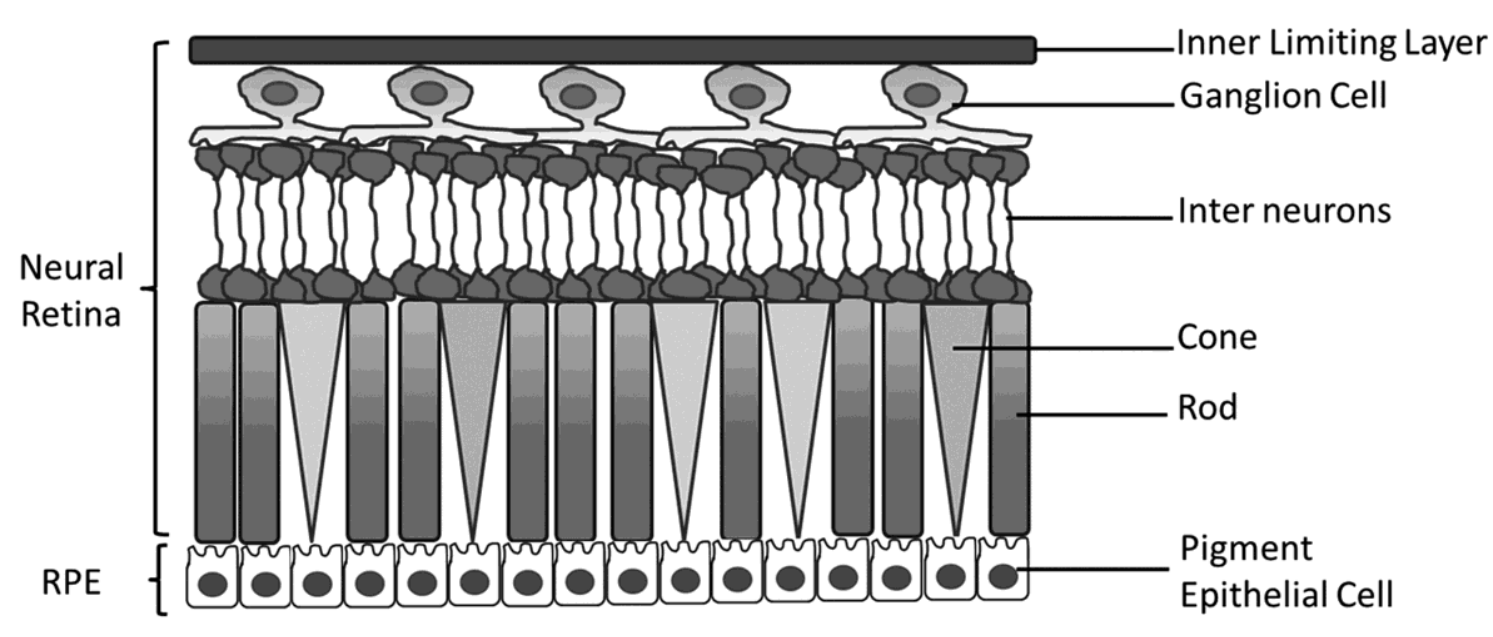

A)

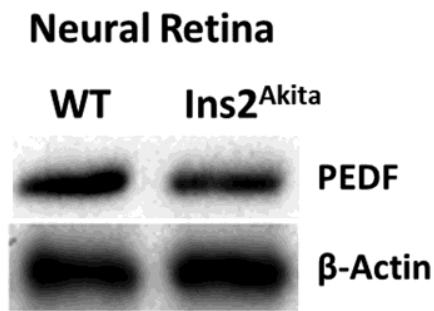

B)

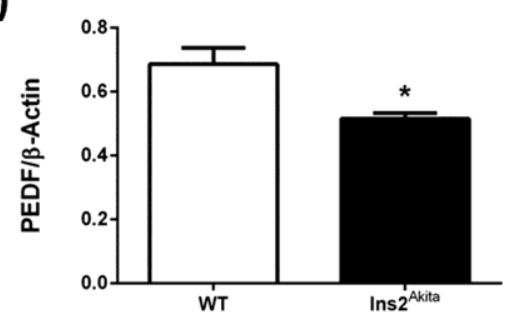

C)

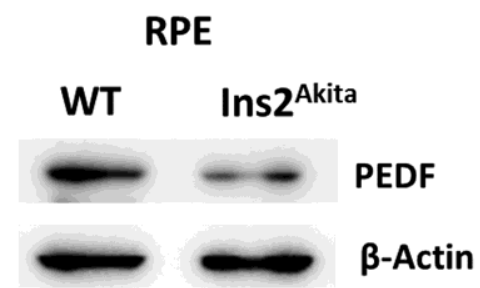

D)

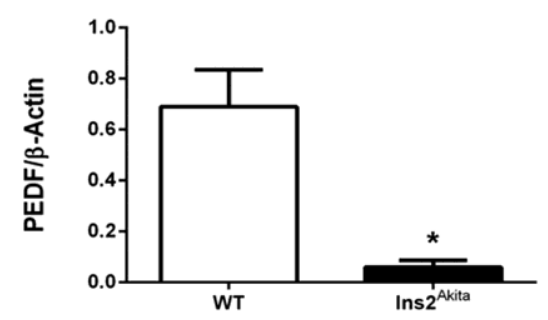

Figure 1: PEDF expression is significantly reduced in the retina of diabetic mice. PEDF protein expression was assessed by Western blot of inner retina (A) and RPE (C) tissue from diabetic and wild type (WT) mice. B and $\mathbf{C}$ represent quantitative data normalized by the intensity of $\beta$-Actin bands. $N=5,{ }^{*} P<0.05$ represents significant differences in PEDF expression in the retina of Ins2 ${ }^{\text {Akita }}$ mice compared with the age-matched WT mice, determined by the two-tailed t-test. 
Page 18 of 24 


\section{Figure 2}

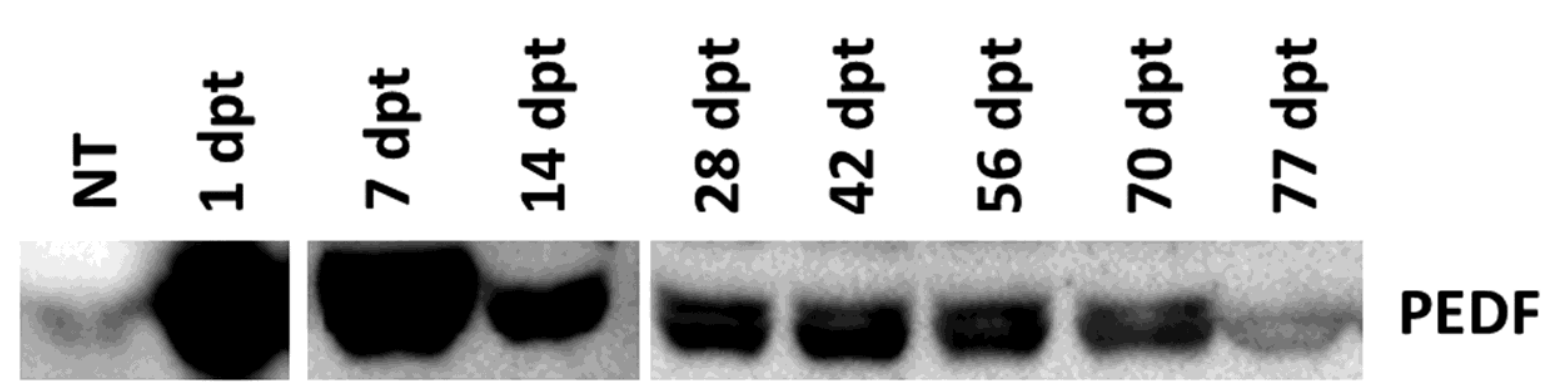

Figure 2: Long-term expression of PEDF by D407 cells transfected with pEPito-hCMVPEDF. pEPito-hCMV-PEDF was able to overexpress PEDF for up to $77 \mathrm{dpt}$ (days posttransfection) in mitotically active cells in levels significantly higher than those observed for non-transfected cells (NT). 
Figure 3

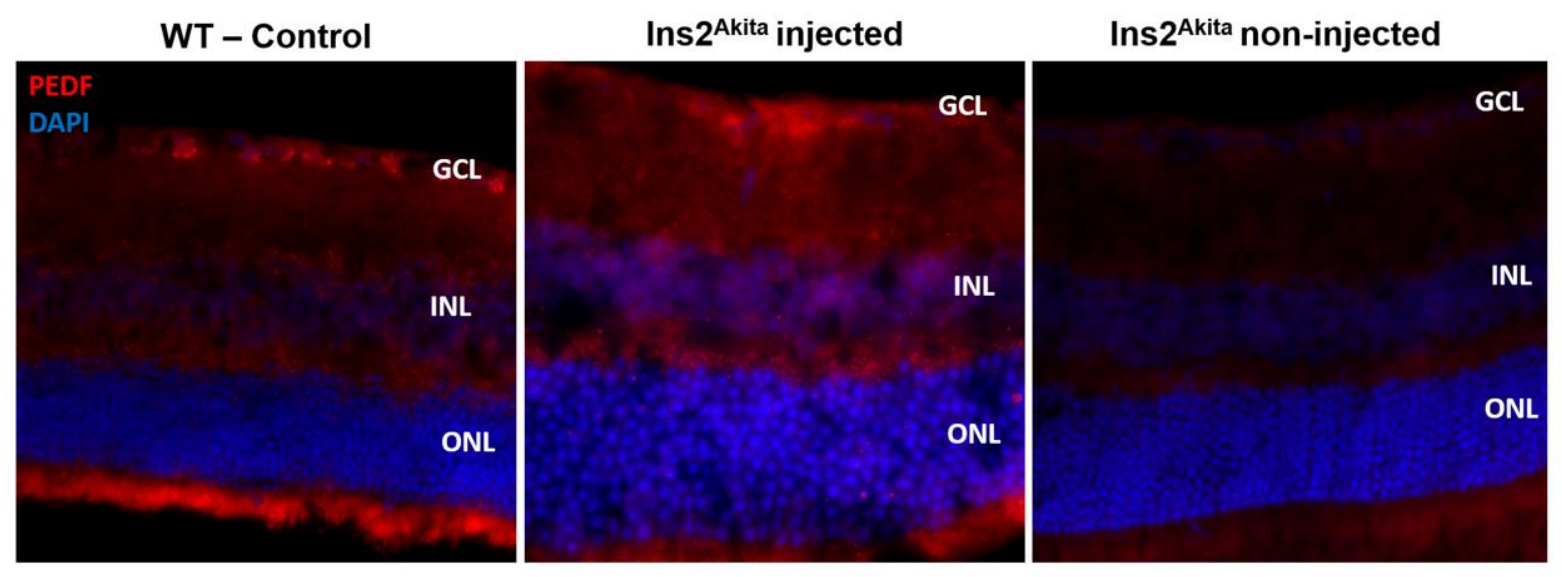

Figure 3: PEDF protein levels are restored after injection of pEPito-hCMV-PEDF. Retinal section of an Ins2 ${ }^{\text {Akita }}$ mouse eye injected with pEPito-hCMV-PEDF-IB three months post injection, showing PEDF levels comparable to age-matched WT mice. The contralateral, non-injected eye, exhibits a significant reduction in PEDF expression. DAPI (blue) stains nuclei and PEDF is marked red. Magnification: 400x 


\section{Figure 4}

\section{Neural Retina}
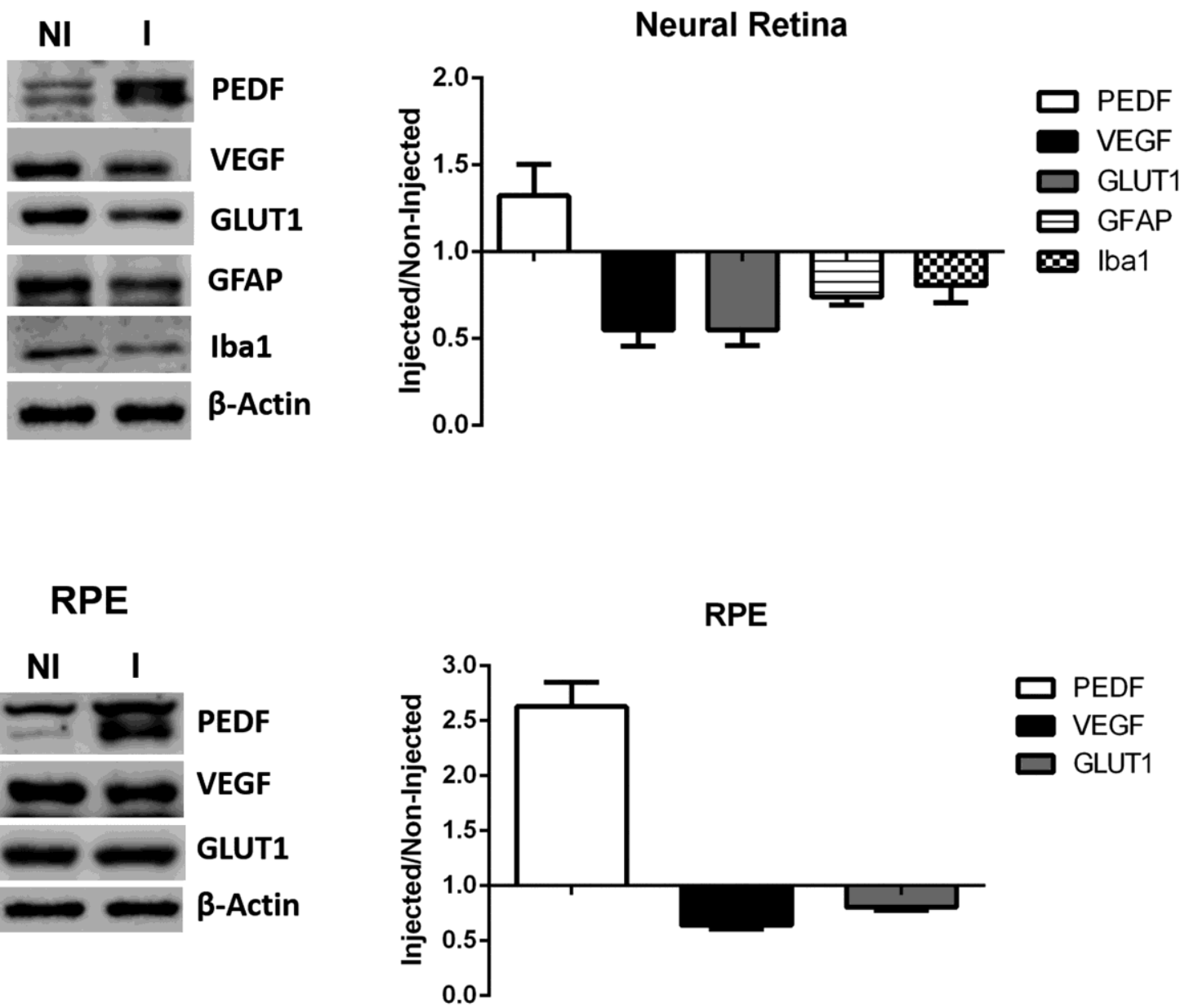

Figure 4: Overexpression of PEDF driven by pEPito-hCMV-PEDF three months post subretinal injection in Ins2 $2^{\text {Akita }}$ mice. Expression of several markers relevant for the DR phenotype was analyzed in whole retinas and RPE cells. PEDF expression was significantly higher in injected animals, while VEGF (angiogenic factor), GLUT1 (responsible for glucose uptake to the cell), Iba1 and GFAP (marker of microglia reactivity and indicator of inflammation, respectively) show reduction of their levels. Quantification data was obtained by normalization with loading control $\beta$-Actin bands, followed by a ratio between the expression of each marker in the injected eye (I) over the non-injected (NI) one. 
Page 22 of 24

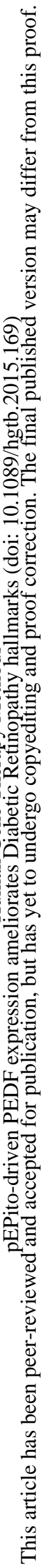


Figure 5

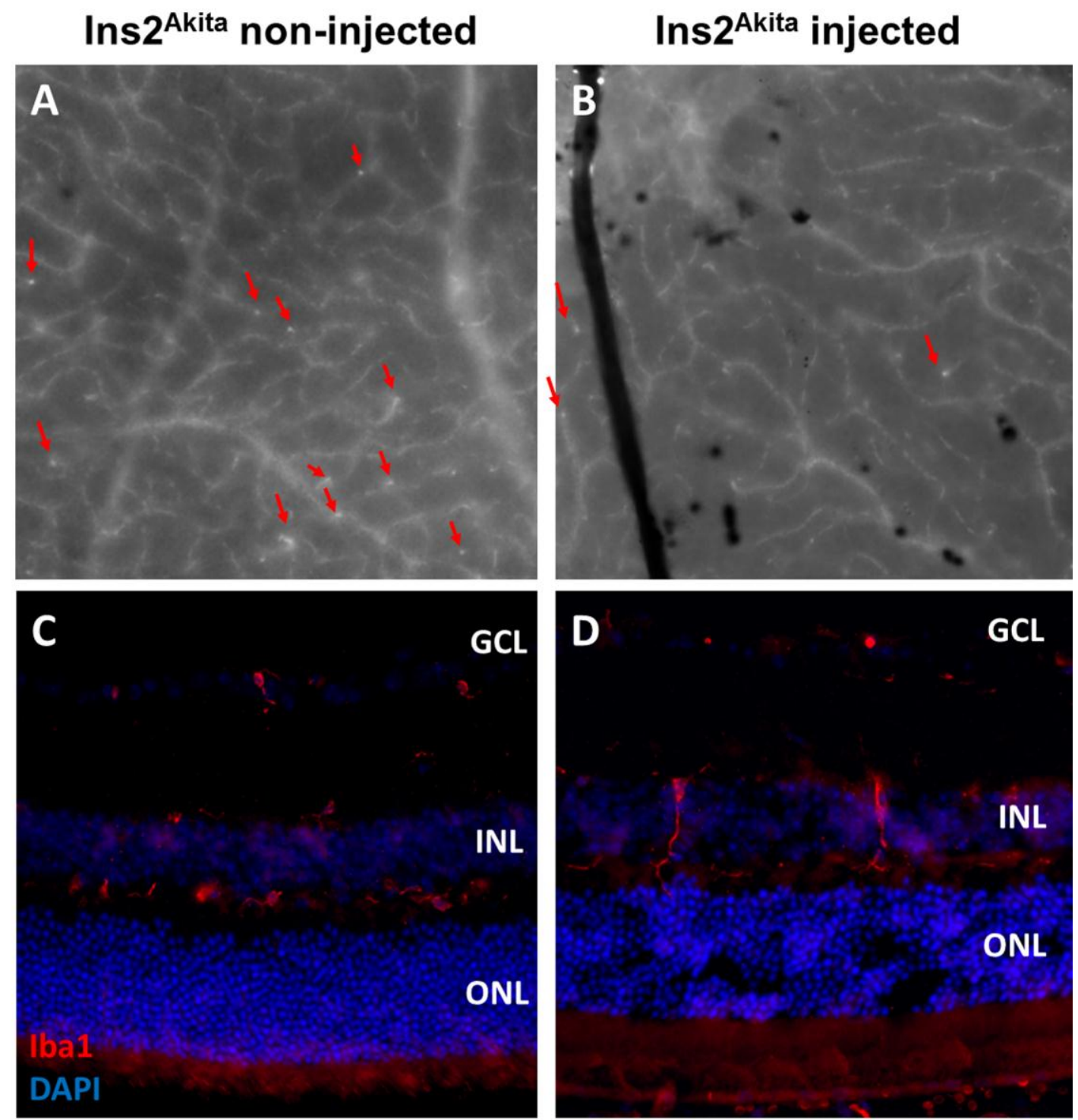

Figure 5: Overexpression of PEDF reduces microglia reactivity in the retina of diabetic mice. Whole mount retinas (upper panels, A and B) and section of an Ins2 ${ }^{\text {Akita }}$ mouse eye (lower panels, $C$ and D) staining with Iba1. Panels of pEPito-hCMV-PEDF injected retinas three months post injection show migroglia with non-reactive morphology. Non-injected retinas display retracted and swollen projections, typical of reactive microglia. DAPI (blue) stains nuclei and Iba1 is stained in red. Magnification: 400x 
Page 24 of 24 